activity, pain intensity, worse fatigue, global health and function were observed among patients with CWP compared to those with NCP. There was a trend towards lower pain tolerance in patients with CWP compared to NCP (Table). Lower pain tolerance and higher TSI scores were observed among patients reporting VAS pain $>40$ versus those with VAS pain scores $\leq 40$ ((mean (SD)) 51.9 (21.2) versus 68.1 (28.1), $p=0.007 ; 0.73(0.60)$ versus $0.55(0.59), p=0.045)$. Conclusions: In this population-based, cross-sectional study of established ax-SpA, chronic widespread pain was common, affecting $50 \%$ of the patients and generally associated with higher disease activity and worse function. CPA shows promising results regarding assessment of pain sensitivity, although larger studies are needed for more conclusive results.

Acknowledgements: Miriam Walsh-Ingelström performing all CPA assessments Disclosure of Interest: E. Mogard: None declared, T. Olofsson: None declared, A. Bremander: None declared, S. Bergman: None declared, L.-E. Kristensen Grant/research support from: Oak Foundation, Consultant for: AbbVie, Celgene, BMS, MSD, Novartis, Pfizer, UCB, J. Kvistgaard Olsen: None declared, J. Wallman Consultant for: Celgene, Novartis, UCB, E. Lindqvist: None declared DOI: 10.1136/annrheumdis-2017-eular.4626

\section{FRI0437 EFFECTS OF ANKYLOSING SPONDYLITIS ON PLANTAR PRESSURE DISTRIBUTION}

E. Mesci ${ }^{1}, \mathrm{~N}$. Mesci $^{2}$, A. İçağasıoğlu ${ }^{1} .{ }^{1}$ Physical Therapy and Rehabilitation, Istanbul Medeniyet University Göztepe Education and Research Hospital; ${ }^{2}$ Physical Therapy and Rehabilitation, Haydarpaşa Numune Education and Research Hospital, Istanbul, Turkey

Background: It is considered that postural changes that occur in patients with ankylosing spondylitis (AS) are biomechanically compensated for by contribution from movements of hip, knee and ankle joints [1]. The effects of this phenomenon on the foot, the most distal segment of the kinetic chain, have not been fully elucidated.

Objectives: In the present study, our aim was to investigate possible changes in plantar load distribution in AS patients.

Methods: The study enrolled 30 AS patients diagnosed as per modified New York criteria and 30 healthy controls matched for age, gender and body mass index. Bath Ankylosing Spondylitis Metrology Index (BASMI), Bath Ankylosing Spondylitis Disease Activity Index (BASDAI) and Bath Ankylosing Spondylitis Functional Index (BASFI) scores were obtained for all patients. Using a foot pressure platform, the distance from the center of pressure (CoP) to the posterior heel line was measured in centimeters $(\mathrm{cm})$ in both groups (DCoP) (figure 1). Percent total plantar load distribution over the proximal half and distal half of the foot was calculated.

Results: Mean DCoP distance was significantly shorter in AS patients $9.65 \pm 0.96$ $\mathrm{cm})$ in comparison to that of control group $(10.41 \pm 0.89 \mathrm{~cm})(p=0.011)$. While the percent total plantar pressure applied on proximal half of the foot was $60.46 \pm 7.62 \%$ in AS patients, it was only $55.95 \pm 6.21 \%$ in the control group $(p=0.031)$. However, AS patients had less percent pressure applied on the distal half of the foot $(39.53 \pm 7.62 \%)$ versus control patients $(44.04 \pm 6.21 \%)(p=0.031)$. The mean ratio of the percent pressure distribution over the proximal half of the foot to the percent pressure distribution over the distal half of the foot was significantly greater in AS patients $(1.64 \pm 0.62)$ compared to control group $(1.31 \pm 0.35)(p=0.037)$.

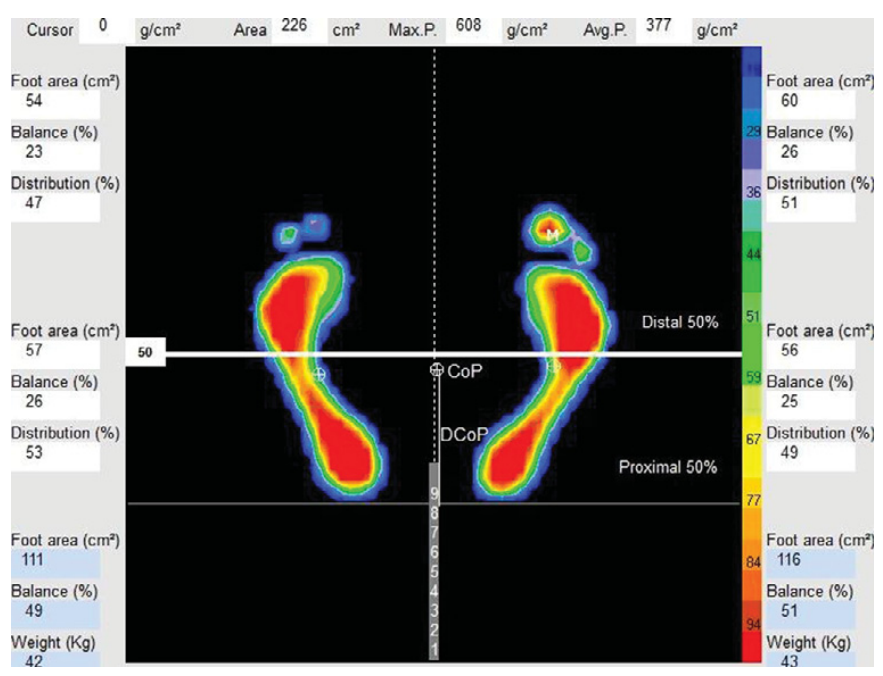

Conclusions: Plantar pressure distribution seems to be displaced towards the heel in the sagittal plane due to biomechanical changes in patients with ankylosing spondylitis. This points out to the significance of the lower extremities in the compensation of postural changes in AS patients.

References:

[1] Murray HC, Elliott C, Barton SE, Murray A: Do patients with ankylosing spondylitis have poorer balance than normal subjects? Rheumatology 2000 , 39:497-500.

Disclosure of Interest: None declared

DOI: 10.1136/annrheumdis-2017-eular.6241

\section{FRI0438 RISK FACTORS FOR UVEITIS IN PATIENTS WITH ANKYLOSING SPONDYLITIS: A RETROSPECTIVE COHORT STUDY}

E.E. Lee, M.J. Kim, E.Y. Lee, Y.W. Song, E.B. Lee. Division of Rheumatology, Department of Internal Medicine, Seoul National University Hospital, Seoul, Korea, Republic Of

Background: The uveitis is one of the most common extra-articular manifestation of ankylosing spondylitis (AS), occurring in $20-30 \%$ of the patients with AS. But it is not clear which factors are associated with the occurrence of uveitis in patients with AS.

Objectives: To evaluate the risk factors for uveitis in patients with AS.

Methods: A total of 1061 Korean patients who were diagnosed with AS at Seoul National University Hospital between January 2004 and December 2015 were evaluated in this study. Using electronic medical record review, the information on the occurrence of uveitis after AS diagnosis and the other demographic and clinical characteristic were obtained, which included age, gender, disease duration, previous history of uveitis, smoking status, Bath ankylosing spondylitis activity index, HLA-B27 positivity and level of serum inflammatory markers. After dividing the patients into those who experienced uveitis after AS diagnosis (uveitis group) and those who did not (non-uveitis group), the potential risk factors for the occurrence of uveitis were evaluated using univariable and multivariable logistic regression analysis. Receiver operating curve analysis was performed.

Results: Among the 1061 patients, 837 (78.9\%) were male and their mean (SD) age was $27.9(6.2)$ years. During the observation of 4231 patient-year, uveitis occurred in 142 (13.4\%) patients (uveitis group). Patients in the uveitis group had longer disease duration than those in the non-uveitis group $(10.0 \pm 6.6$ vs. $8.2 \pm 6.2$ years, $p=0.003$ ). The proportion of the patients with prior uveitis before diagnosis of AS or HLA-B27 positivity were higher in the uveitis group than those in the non-uveitis group. ( $58.5 \%$ vs. $12.1 \%, 95.7 \%$ vs. $83.7 \%$, respectively) There were no significant differences in ESR and CRP between the two groups. In the multivariable analysis, two most significant risk factors for new-onset uveitis was the prior uveitis before the diagnosis of $A S(O R=11.18[95 \%$ confidence interval (Cl): 7.09-17.63]) and HLA-B27 positivity (OR=9.15 [95\% Cl: 1.23-68.41]). The area under the curve (AUC) of the final multivariable model was $0.809(95 \% \mathrm{Cl}$ : 0.77-0.85).

Table 1. Risk factors for uveitis in patients with AS $(n=1061)$

\begin{tabular}{lccc}
\hline & Uveitis group $(\mathrm{n}=142)$ & Non-uveitis group $(\mathrm{n}=919)$ & $\mathrm{p}$-value \\
\hline Males, $\mathrm{n}(\%)$ & $104(73.2)$ & $733(79.8)$ & 0.076 \\
Age on symptom onset (years) & $28.51 \pm 11.56$ & $27.82 \pm 11.95$ & 0.541 \\
Disease duration (years) & $10.03 \pm 6.60$ & $8.22 \pm 6.21$ & ${ }^{*} 0.003$ \\
History of uveitis, $\mathrm{n}(\%)$ & $83(58.5)$ & $111(12.1)$ & ${ }^{*}<0.001$ \\
Smoking, $\mathrm{n}(\%)$ & $35(25.0)$ & $184(20.3)$ & 0.086 \\
ESR $(\mathrm{mm} / \mathrm{h})$ & $38.84 \pm 31.07$ & $28.10 \pm 27.33$ & ${ }^{*} 0.001$ \\
CRP $(\mathrm{mg} / \mathrm{L})$ & $1.93 \pm 2.70$ & $1.60 \pm 2.61$ & 0.216 \\
HLA-B27, $\mathrm{n}(\%)$ & $135(95.7)$ & $764(83.7)$ & ${ }^{*}<0.001$ \\
${ }^{+}$BASDAI & $6.44 \pm 1.71$ & $6.59 \pm 1.68$ & 0.500 \\
\hline
\end{tabular}

${ }^{\dagger}$ BASDAI in AS patients who exposed to TNF inhibitors.

Multivariate analysis showed that history of uveitis and the presence of HLA-B27 as the final risk factors for the occurrence of uveitis.

Conclusions: Prior uveitis before the diagnosis of AS and HLA-B27 positivity were associated with the occurrence of uveitis. These results can be useful to predict occurrence of uveitis in patients with AS.

Disclosure of Interest: None declared

DOI: 10.1136/annrheumdis-2017-eular.2943

\section{FRI0439 PSYCHIATRIC DISORDERS ASSOCIATED WITH ANKYLOSING SPONDYLITIS}

F.G. Yurdakul, Y. Garip, R. Koçak Ulucaköy, E. Almaz, A. Uçkun, H. Bodur. Physical Medicine and Rehabilitation, Ankara Numune Training and Research Hospital, Ankara, Turkey

Background: Ankylosing spondylitis (AS) is an inflammatory rheumatic disease characterized by spinal and/or peripheral involvement, enthesitis, dactylitis, and several extra-articular manifestations. Chronic inflammation often leads to reduced spinal mobility and functional disability. The frequency of fatigue, sleep disturbance, and psychological problems has increased in AS patients $(1,2)$.

Objectives: Although there are studies investigating depression and anxiety frequency in AS patients, different psychiatric disorders such as impulsivity, alexithymia and eating disorders have not been evaluated. The aim of this study is to investigate the frequency of different psychiatric disorders in AS patients, and to evaluate the relationship between these disorders with disease activity and functional status.

Methods: Patients with AS $(n=70)$ and healthy controls $(n=56)$ were included in the study. The Ankylosing Spondylitis Disease Activity Score (ASDAS), Bath Ankylosing Spondylitis Functional Index, Bath Ankylosing Spondylitis Metrology 